\title{
Molecular dynamics study of nanoconfined TIP4P/2005 water: how confinement and temperature affect diffusion and viscosity
}

\author{
A Zaragoza ${ }^{1,2}$, MA Gonzalez ${ }^{3}$, L Joly ${ }^{4}$, I Lopez-Montero ${ }^{3}$, MA Canales $^{5}$, AL Benavides $^{2}$, and C Valeriani ${ }^{1}$ \\ ${ }^{1}$ Departamento de Estructura de la Materia, Facultad de Ciencias F'sicas, \\ F'sica Térmica y Electrónica, Universidad Complutense de Madrid, 28040 Madrid, Spain. \\ 2 Depto. Ingenier'a F'sica, División de Ciencias e Ingenier'as, \\ Universidad de Guanajuato, 37150 León, Mexico. \\ 3 Departamento de Qu'mica F'sica, Facultad de Ciencias Qu'micas, \\ Universidad Complutense de Madrid, 28040 Madrid, Spain. \\ ${ }^{4}$ Univ. Lyon, Université Claude Bernard Lyon 1, CNRS, \\ Institut Lumière Matière,F-69622 Villeurbanne, France. \\ ${ }^{5}$ Instituto de Investigación Hospital Doce de Octubre (i+12), Avenida de Cérdoba s/n, 28041 Madrid, Spain. \\ ${ }^{6}$ Departamento de Qu'mica Orgánica I, Facultad de Ciencias Qu'micas, \\ Universidad Complutense de Madrid, 28040 Madrid, Spain.
}

(Dated: December 23, 2019)

\begin{abstract}
In the last decades a large effort has been devoted to the study of water confined in hydrophobic geometries at the nanoscale (tubes, slit pores), because of the multiple technological applications of such systems, ranging from drugs delivery to water desalinization devices. To our knowledge, neither numerical/theoretical nor experimental approaches have so far reached a consensual un- derstanding of structural and transport properties of water under these conditions. In this work, we present molecular dynamics simulations of TIP4P/2005 water under different hydrophobic nanoconfinements (slit pores or nanotubes, with two degrees of hydrophobicity) within a wide temperature range. On the one side, water is more structured near the hydrophobic walls, inde- pendently on the confining geometries. On the other side, we show that the combined effect of confinement and curvature leads to an enhanced diffusion coefficient of water in hydrophobic nan- otubes. Finally, we propose a confined Stokes-Einstein relation to extract viscosity from diffusivity, whose result strongly differs from the Green-Kubo expression that has been used in previous work. We discuss the shortcomings of both approaches, which could explain this discrepancy.
\end{abstract}

PACS numbers:

\section{INTRODUCTION}

Water is essential for life. Although it is formed by simple triatomic molecules, it has an unusual behavior when compared with similar mono-component substances.[1] It exhibits many anomalies and a lot of work has been devoted to understand and describe its anomalous behavior. Besides, under confinement, water exhibits a different phase diagram than bulk water, where the anomalies can disappear or occur at different thermodynamic conditions. [2] When confinement reaches the nanoscale, water undergoes novel physical properties, different from their bulk analog and still not completely understood. [37] Research on nano confined water has become of great interest due to its multiple nano technology applications, as for instance cells biochannels, [8-10] drugs delivering, [11-14] water desalination devices, [15-22] among others. [22-30]

Due to the small length scales, the study of water in nano confinement is experimentally very challenging, as shown by the few experimental works on the subject. $[2,31,32]$ Besides, experimental results are controversial and open problems remain. [33] This is the main reason why there has been a greater interest in using theoretical approaches or molecular simulations to un- derstand, for instance, the effect of varying the geometrical parameters defining the nano confinement and/or the thermodynamic conditions. [24, 34-41] However, to our knowledge, neither the theoretical and simulations approaches, nor the experimental ones, have so far reached a consensus on how water transport properties are affected under these conditions. [33, 42]

Thus, molecular dynamics (MD) simulation is a very important tool to study confined water. A good force field is then required to model the water-water and the water-wall interactions. For pure water simulation studies, many force fields have been proposed to reproduce the complex phase diagram of water and its anomalies;[43] among them one can list: SPC,[45] TIP3P, [44] TIP4P, [44] AMOEBA,[46] and TIP $4 \mathrm{P} / 2005,[47]$ the latter being one of the most robust and accurate. For confined water (between walls, inside pores or nanotubes) using rigid atomistic models, most of simulation works have been performed using TIP3P or SPC/E models and only a few have used the TIP4P/2005 model. [34, 48-60]

A reliable MD simulation study for water transport in carbon nanotubes (CNTs) in order to find out conduction rates with different water models has been done by Liu and Patey [58] with a pressure-driven water transport (non-equilibrium conditions) through $(8,8)$ and $(9,9)$ 
CNTs using three different water models (TIP3P, SPC/E and TIP4P/2005). In a second paper, the same authors have considered smaller tubes and rationalized the differences in terms of viscous entrance effects controlled by the viscosity of the water model at the entrance of the tube. [61]

Among recent simulation works on confined TIP4P/2005 water under equilibrium conditions, one can mention the work of Köhler et al. [48] and Marti et al.[49] Both groups considered this model either confined inside or outside CNTs with hydrophobic or hydrophilic walls. Different diameters were considered and their effect on structural or transport properties were analyzed for some selected thermodynamic conditions. They found interesting results, however, since Köhler et al. studied CNTs $(10,10),(16,16)$, and $(30,30)$, meanwhile, Marti et al. CNTs $(5,5),(9,9)$, and $(12,12)$, and the latest authors used different water-carbon interaction models, it is not possible to give a general conclusion of their findings. For instance, Köhler et al. found a great influence of the density, confinement size, and water-wall interaction on the viscosity. Marti et al. [49] besides nanotubes, considered two graphene sheets separated by a distance from $0.6 \mathrm{~nm}$ up to $1.7 \mathrm{~nm}$ immersed in TIP $4 \mathrm{P} / 2005$ water at $\mathrm{T}=275 \mathrm{~K}$ and $\mathrm{p}=400$ bar. They found that the diffusion of water depends on the plates distance in a non-monotonic way. As can be noticed, even for this popular water model under confinement, the available information is limited and the same occurs with the experimental data.

So, in this work we try to cover this gap and have chosen the TIP4P/2005 water model confined between rigid hydrophobic carbon parallel walls, separated by a distance $1.6 \mathrm{~nm}$ and $5.6 \mathrm{~nm}$, and inside CNTs (20,20), $(35,35)$ and $(52,52)$. This study is carried out using MD simulations, underlying the effect (if any) played by the hydrophobicity of the surface and by the temperature of the system. Our study aims at being a benchmark for future works, explaining quantitatively the water structure under confinement, its dynamics and the number of hydrogen bonds per molecule formed inside these configurations. Additionally, in this work a few NMR experimental measurements to predict the diffusion coefficient of water in CNTs are presented and compared with available experimental data.

The manuscript is organized as follows: In Section 1, the TIP4P/2005 water model and the water-carbon hydrophobic interactions are discussed. In Section 2, we present the simulation details as well as those of the nanostructures to be considered and implemented in simulations. In Section 3, simulation results for the structural properties (density profiles and hydrogen-bonds distributions) and transport properties (diffusion coefficient and viscosity) are given for different confinement geometries and temperatures. For viscosity, we derived a confined Stokes-Einstein formula, which we compare to the commonly used Green-Kubo formula. Finally, the main conclusions of this work are given in section 4. This article is complemented with three appendixes. Appendix A describes a drop-shape analysis to show how we selected the water-wall interaction. In Appendix B, a confined Stokes-Einstein relation is derived for a liquid confined between parallel stress-free walls, in the framework of continuum hydrodynamics. Appendix C contains our experimental attempt of measuring the diffusion coefficient of water confined in CNTs using Nuclear Magnetic Resonance (NMR) technique.

\section{SYSTEMS AND METHODS}

Throughout this work, we have carried out NVT simulations using the Molecular Dynamics package GROMACS 2016.4,[62] for water confined under two geometries: between two parallel graphene walls (W) and inside carbon nanotubes (CNT). We made this study for several temperatures in the range $243 \mathrm{~K}$ to $298 \mathrm{~K}$. We set the timestep to $1 \mathrm{fs}$ and simulate every temperature for at least $40 \mathrm{~ns}$, and for lowest temperatures (243-253 K) up to $60 \mathrm{~ns}$. In order to keep the temperature constant, we use a Canonical sampling through velocity rescaling thermostat [63] with a relaxation time set to $1 \mathrm{ps}$. We checked that the same results were obtained by means of Berendsen or Nosé-Hoover thermostats. We are aware of the fact that thermostating confined fluids is a very delicate issue [64-67] given that there is no perfect way to thermostat a confined liquid. However, what we have used in our work is not an uncommon approach. We have checked different damping times (1ps being a long relaxation time), and thermostats to make sure that the chosen thermostat was not affecting our results.

We used a block average method to estimate the error bars [68].

\section{A. Tuning the hydrophobicity of the interaction potential}

Water molecules have been simulated using the TIP4P /2005 force field [47]. We truncated the LennardJones (LJ) potential at $9.5 \AA$, adding standard tail corrections to the LJ energy, and considered Ewald sums (with the PME technique) [69] for the calculation of the longrange electrostatic forces, applying a real space cut-off at $9.5 \AA$. The carbon-oxygen (CO) diameter interaction is described by a geometric average, $\sigma_{C O}=\sqrt{\sigma_{C C} \sigma_{O O}}$, where $\sigma_{C C}$ is taken from the OPLS-AA force field [70]. The value of $\varepsilon_{C O}$ established the amount of hydrophobicity of the carbon wall. In this work we have used a value of $\varepsilon_{C O}$ widely used for water in graphite confinement $[21,48,71,72]$ correponding to a contact angle of around $96.5^{\circ}$. For comparison, we have also used a superhydrophobic water-carbon interaction value previouly 
TABLE I: TIP4P/2005 water model force field parameters. A Lennard-Jones potential characterizes the oxygen-oxygen, carbon-carbon (OPLS-AA) and carbon-oxygen interactions $\left(\varepsilon_{c o}^{h}\right.$ and $\left.\varepsilon_{c o}^{s h}\right)$. The charge $\left(\mathrm{q}_{H}\right.$ and $\mathrm{q}_{M}$ are the hydrogen and dummy atom charges respectively) and angle refer to TIP4P/2005 water model.

\begin{tabular}{|c|c|c|}
\hline$\overline{\overline{\text { Interaction }}}$ & $\overline{\overline{\sigma(\mathrm{nm})}}$ & $\overline{\overline{\varepsilon(\mathrm{kJ} / \mathrm{mol})}}$ \\
\hline \multicolumn{3}{|c|}{ Lennard-Jones parameters } \\
\hline$\overline{\mathrm{O}-\mathrm{O}}$ & 0.3159 & 0.7749 \\
\hline C-C & 0.3550 & 0.2929 \\
\hline $\mathrm{C}-\mathrm{O}(\mathrm{h})$ & 0.3349 & 0.2703 \\
\hline $\mathrm{C}-\mathrm{O}(\mathrm{sh})$ & 0.3349 & 0.0472 \\
\hline \multicolumn{3}{|c|}{ Charges (e) } \\
\hline $\mathrm{q}_{H}=-\mathrm{q}_{M} / 2$ & & 0.5564 \\
\hline \multicolumn{3}{|c|}{$\overline{\mathrm{H}_{2} \mathrm{O} \text { angle (degrees) }}$} \\
\hline$\overline{\angle \mathrm{H}_{2} \mathrm{O}}$ & & $104.52^{\circ}$ \\
\hline
\end{tabular}

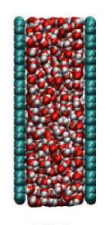

W-1

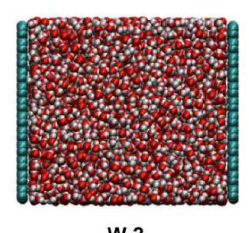

W-2

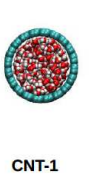

CNT-1

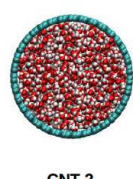

CNT-2

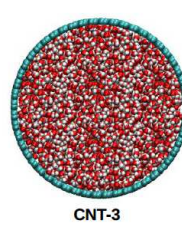

CNT-3
FIG. 1: Snapshots of the nano-structures used in this work, made of carbon atoms (cyan spheres), filled with water molecules (red for oxygens and white for hydrogens). Confined systems between graphene walls are labeled as "W", and inside nanotubes as "CNT". Different numbers refer to systems with an increasing wall-distance (W) or diameter (CNT).

used by Algara-Siller et al. in Ref. [23] corresponding to a higher contact angle, $132^{\circ}$ and $\varepsilon_{c o}^{s h}=0.0476 \mathrm{~kJ} / \mathrm{mol}$. As detailed in Appendix A, the contact angles were estimated by simulating a sessile (nano)droplet of water on planar walls. In Table I, we report the parameters of the force fields used for water and carbon.

\section{B. System preparation}

To start with, we prepare the general geometries filled with water molecules as shown in Fig. 1. Carbon atoms are placed on a lattice of graphene with fixed positions. Concerning nanotubes, atoms are placed according to the chirality specified in each case, also with fixed positions (frozen). The Carbon-Carbon interaction parameters $(\varepsilon$ and $\sigma$ ) are presented in table I. These values correspond to OPLS-AA force-field as it was reported in reference [70].

The $\mathrm{W}$ geometry consists of two $5.2 \mathrm{~nm} \times 5.2 \mathrm{~nm}$ parallel graphene walls, each made of 1008 carbon atoms (1
TABLE II: Top: Numerical details for the W-type confinement: number of carbon atoms per wall $\left(\mathrm{N}_{C}\right)$, distance between parallel walls (d), wall's edge (corresponding to the simulation box limit, $\left.\mathrm{L}_{\mathrm{Box}}\right)$ and number of water molecules $\left(\mathrm{N}_{W}\right)$. Bottom: Numerical details for the CNT-type confinement: chirality, number of carbon atoms per nanotube, nanotube diameter, nanotube length (corresponding to the simulation box edge) and number of water molecules.

\begin{tabular}{lccccc}
\hline \hline System & $d(\mathrm{~nm})$ & $L_{\text {Box }}(\mathrm{nm})$ & $\mathrm{N}_{C}$ (per wall) & $\mathrm{N}_{w}$ \\
\hline $\mathrm{W}-1$ & 2.0 & 5.1 & \multicolumn{2}{c}{1008} & 1361 \\
$\mathrm{~W}-2$ & 6.1 & 5.2 & \multicolumn{1}{c}{1008} & 5417 \\
\hline \hline & & & & & \\
\hline \hline System & $(n, m)$ & $d(\mathrm{~nm})$ & $L_{\text {Box }}(\mathrm{nm})$ & $\mathrm{N}_{C}$ & $\mathrm{~N}_{w}$ \\
\hline CNT-1 & $(20,20)$ & 2.6 & 5.16 & 1680 & 640 \\
CNT-2 & $(35,35)$ & 4.7 & 5.15 & 2940 & 2242 \\
CNT-3 & $(52,52)$ & 7.0 & 6.20 & 5201 & 6407 \\
\hline \hline
\end{tabular}

atom thick). In order to be able to apply periodic boundary conditions in all directions, the system, i.e. water and carbon walls, is located at the center of the simulation box and an empty region is left between the parallel walls and the system's periodic replicas (as suggested in Ref. 38). We prepare two setups: W-1, containing 1361 water molecules confined between hydrophobic walls located at a distance of $1.6 \mathrm{~nm}$; and W-2 containing 5417 water molecules confined between hydrophobic walls located at a distance of $5.6 \mathrm{~nm}$.

The CNT geometry consists of cylindrical hydrophobic nanotubes of the following chirality: $(20,20)$ (CNT-1), $(35,35)(\mathrm{CNT}-2)$ and $(52,52)$ (CNT-3). We use the Visual Molecular Dynamics software [73] to prepare every nanotube. A single wall CNT is centered in the middle of the simulation box. Periodic boundary conditions are applied in all directions, but the interactions between the system and its replicas along $\mathrm{X}$ and $\mathrm{Y}$ are avoided by using very large simulation box sizes as it was shown by Zheng et al. [54] Numerical details of these setups are reported in Table II.

To establish the density of confined water, we compute it as the number of water molecules divided by the volume that they are contained in, $\mathrm{N}_{w} / \mathrm{V}$ ( $V$ being either the volume between the two walls or the volume inside the nanotube). A recipe to estimate the volumes for the two geometries will be given in the Results section. For ultra-confined systems this could make a difference as compared to fixing the pressure. Whereas here the systems are above the critical size where sub-continuum behaviour is expected. [74]

\section{Measured quantities}

In order to give a molecular explanation of water behavior under nano-confinement, we firstly computed the density profile of water molecules and calculated the average number of hydrogen bonds (HBs) per molecule. The 
HB criterion is based on the number of donor hydrogenbonds per molecule as proposed by Kumar et al. [75], where the HB distance and angle necessary to bond two water molecules are $d \approx 0.28 \mathrm{~nm}$ and $\widehat{O H}=109.4^{\circ}$.

Next, we computed the viscosity of confined water which can be a delicate issue in confined systems. [7680]

In a bulk homogeneous liquid, viscosity can be computed by means of the Green-Kubo expression:

$$
\eta_{\mathrm{GK}}=\frac{V}{k_{B} T} \int_{0}^{\infty} d t\left\langle P_{\alpha \beta}(t) P_{\alpha \beta}(0)\right\rangle,
$$

where $\mathrm{P}_{\alpha \beta}$ are the traceless components of the pressure tensor. In a confined liquid however, the system becomes heterogeneous, and more important, the thermal fluctuations of the shear stress at equilibrium will not only be affected by the liquid viscosity, but by the slip boundary condition at the wall; consequently, the Green-Kubo formula provides only an effective viscosity whose physical meaning is unclear. Nevertheless, it can be interesting to compute $\eta_{\mathrm{GK}}$ and to compare it with other possible measurements of confined viscosity, as discussed later. Note also that in confined systems, the volume $V$ of the liquid is not well defined, with a related uncertainty on the computed $\eta_{\mathrm{GK}}$, and that the stress tensor components $P_{\alpha \beta}$ depend on the system's geometry. Therefore, we must distinguish between axial viscosity (defined by axial pressure components $\mathrm{P}_{x y}$ ) and radial viscosity (defined by radial components $\mathrm{P}_{x z}$ and $\mathrm{P}_{y z}$ ), as proposed by Kölher et al. In this work, we have calculated for the two different types of confinement the axial viscosity, integrating the autocorrelation function of the axial components.

Another approach to estimate the viscosity relies on computing the self-diffusion coefficient and using a confined Stokes-Einstein relation. The diffusion coefficient $D_{\|}$under confinement can be measured using the particle's mean square displacement at long time:

$$
D_{\|}=\frac{\left\langle\left|r\left(t_{0}+t\right)-r\left(t_{0}\right)\right|^{2}\right\rangle}{2 \operatorname{dim} \times t}
$$

where dim depends on the system's geometry: being $\operatorname{dim}=1$ for CNT and $\operatorname{dim}=2$ for parallel walls.

Diffusion measurements in bulk liquids via MD are strongly affected by finite size effects due to hydrodynamic interactions with periodic images of the simulation box [81-84]. Analytical corrections exist both for isotropic or anisotropic simulation boxes. However, the case of confined systems is quite different and has been less explored. In planar confinement, assuming a no-slip boundary condition on the walls, Simonnin et al.[85] have computed analytically the effect of liquid height $d$ and box lateral size $L$ on the diffusion coefficient. Here we would like to emphasize that, while the effect of the finite lateral size $L$ is purely a limit of the simulation, the confinement height $d$ has a real physical effect. Indeed, diffusion is affected in the vicinity of walls. Analytical descriptions can be found for planar confinement following Faxen's pioneering work [86]; in particular, it has been shown that liquid-solid slip impacts how diffusion is affected close to walls. [87-89] Regarding the effect of the lateral size $L$, the exact formula derived by Simonnin et al. [85] can be approximated by assuming that the particle diffusion coefficient is the sum of their intrinsic diffusion coefficient and the diffusion coefficient of the liquid center of mass (com), for which analytical predictions exist in the presence of liquid-solid slip. [90, 91] In practice, this also means that removing the liquid com motion when computing the particle mean square displacement - as we did in this work - provides a good estimate of the intrinsic diffusion coefficient for infinite lateral size $L$. Then only the physical effect of the confinement remains. Simonnin et al. derived an expression for the average parallel diffusion coefficient $\left\langle D_{\|}\right\rangle$in the no-slip case, using Einstein relation between mobility and diffusivity [92], and a continuum hydrodynamics description of Stokes drag in confinement. In graphite confinement, slip is very large, with slip lengths typically much larger than the confinement. [93-96] It is therefore reasonable to assume a perfect slip boundary condition (stress-free, infinite slip length). Under those conditions, and for moderate planar confinement, one can adapt the calculation by Simonnin et al. (see Appendix B) to show that:

$$
\left\langle D_{\|}\right\rangle \approx \frac{k_{\mathrm{B}} T}{3 \pi \eta \sigma_{\mathrm{h}}}\left[1+\frac{3}{8} \frac{\sigma_{\mathrm{h}}}{d} \ln \left(\frac{2 d}{\sigma_{\mathrm{h}}}\right)\right],
$$

with $\eta$ the viscosity (assumed homogeneous and isotropic) and $\sigma_{\mathrm{h}}$ the effective hydrodynamic diameter of the particles [98], which can be computed from bulk measurements of diffusion and viscosity (see Appendix B). In the following, we shall refer to Eq. (3) as the confined Stokes-Einstein relation. Regarding CNT systems, we are not aware of an expression equivalent to Eq. (3) in cylindrical geometry. As a very rough estimate, we suggest to use the same equation, replacing the slab height by the tube diameter. The confined Stokes-Einstein relation provides an alternative estimate of the viscosity:

$$
\eta_{\mathrm{SE}} \approx \frac{k_{\mathrm{B}} T}{3 \pi \sigma_{\mathrm{h}}\left\langle D_{\|}\right\rangle}\left[1+\frac{3}{8} \frac{\sigma_{\mathrm{h}}}{d} \ln \left(\frac{2 d}{\sigma_{\mathrm{h}}}\right)\right],
$$

which we will compare to the Green-Kubo estimate in the results section. Equation (4) provides an effective viscosity, averaged in space and over the different traceless components of the pressure tensor; however, unlike with the Green-Kubo formula, here the effect of the slip boundary condition on the walls is taken into account and separated from the intrinsic viscosity of the confined liquid. Note that the Stokes-Einstein relation is known to break down in supercooled bulk water [97, 98], so that the confined Stokes-Einstein estimate of the viscosity should be taken with caution at very low temperature. 

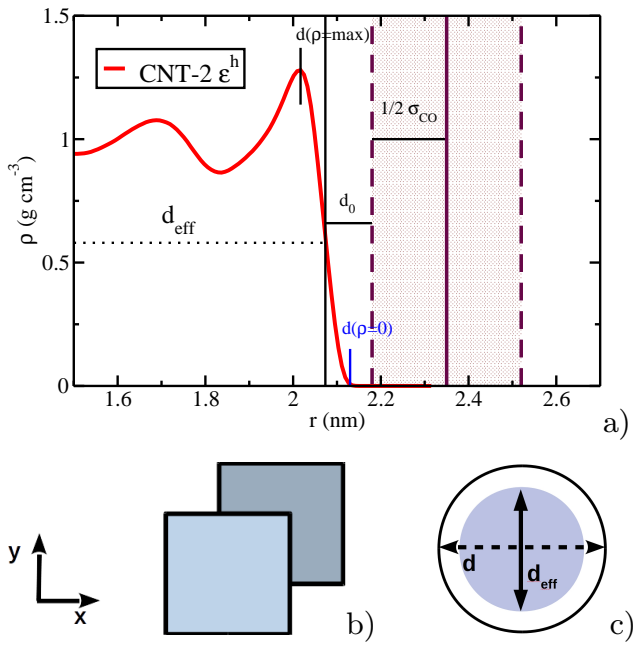

FIG. 2: a) Zoom close to the hydrophobic wall $\left(\varepsilon_{c o}^{h}\right)$ (purple vertical lines) of the water density profiles (red curve) computed from the center of a CNT-2 nanotube at $T=298 \mathrm{~K}$. The meaning of the presented variables is reported in the text. b) and c) are schematic representations of the orientations of both parallel walls (b) and nanotubes (c). In the latter, $d$ indicates the nominal diameter, whereas $d_{\text {eff }}$ indicates the effective one.

\section{RESULTS}

In this section we present our results on water structural (density and HBs) and transport (viscosity and diffusion) properties. We study the effect of hydrophobicity, of nanostructure's curvature (comparing parallel and cylindrical confinement) and of temperature on each of the structural and transport properties.

\section{A. Density profiles}

We computed the density profiles of confined water for every system at several temperatures for both hydrophobic and superhydrophobic interactions. As already observed, the density profile of confined water differs with respect to bulk water, because of curvature effects and water-carbon interaction. [22, 99]

As shown in Fig. 2, when preparing a CNT-2 hydrophobic system containing liquid water with a nominal density of $1 \mathrm{~g} \mathrm{~cm}^{-3}$, the density profile reveals water layering, especially close to the hydrophobic walls. The hydrophobicity of the confining walls expels water molecules of the closest layer, thus reducing the overall confining volume available to the water, which leads to an "effective density" that is higher than the nominal one. To compute the effective volume for the walls and for the nanotubes, the effective distance $d_{\text {eff } f}$ is defined as $d_{\text {eff }}=d-\sigma_{C O}-2 d_{0}$, where $d$ is the nominal wall-to-wall
TABLE III: Nominal distance between walls/the nanotubes diameters $d$, and their effective values $d_{e f f}$, effective volume $V_{e f f}$ and effective density $\rho_{e f f}$ (taking the number of water molecules for each system from Table II).

\begin{tabular}{lcccc}
\hline System & $d(\mathrm{~nm})$ & $d_{\text {eff }}(\mathrm{nm})$ & $V_{\text {eff }}\left(\mathrm{nm}^{3}\right)$ & $\rho_{\text {eff }}\left(\mathrm{g} \cdot \mathrm{cm}^{-3}\right)$ \\
\hline \hline $\mathrm{W}-1 \varepsilon_{c o}^{s h}$ & 2.00 & 1.50 & 38.8 & 1.1 \\
$\mathrm{~W}-2 \varepsilon_{c o}^{s h}$ & 6.10 & 5.60 & 151.4 & 1.1 \\
\hline $\mathrm{W}-1 \varepsilon_{c o}^{h}$ & 2.00 & 1.50 & 38.9 & 1.0 \\
$\mathrm{~W}-2 \varepsilon_{c o}^{h}$ & 6.10 & 5.60 & 151.4 & 1.1 \\
\hline \hline \multicolumn{5}{c}{} \\
\hline \hline $\mathrm{CNT}-1 \varepsilon_{c o}^{s h}$ & 2.60 & 1.97 & 15.73 & 1.2 \\
$\mathrm{CNT}-2 \varepsilon_{c o}^{s h}$ & 4.74 & 4.05 & 66.24 & 1.0 \\
$\mathrm{CNT}-3 \varepsilon_{c o}^{s h}$ & 7.04 & 6.28 & 192.04 & 1.0 \\
\hline $\mathrm{CNT}-1 \varepsilon_{c o}^{h}$ & 2.60 & 2.02 & 16.02 & 1.2 \\
$\mathrm{CNT}-2 \varepsilon_{c o}^{h}$ & 4.74 & 4.20 & 71.23 & 0.9 \\
$\mathrm{CNT}-3 \varepsilon_{c o}^{h}$ & 7.04 & 6.36 & 196.97 & 1.0 \\
\hline \hline
\end{tabular}
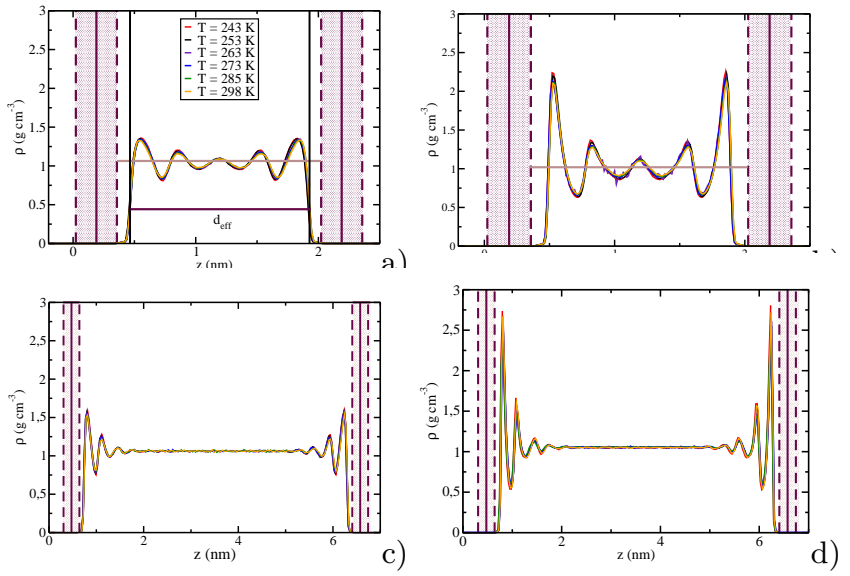

FIG. 3: Density profiles for water confined in W-1 (top panels) and W-2 (bottom panels) geometries at different temperatures (legend shown in figure a)). Results for the superhydrophobic system $\varepsilon_{c o}^{s h}$ are on the left-hand side (a) and (c) whereas for the hydrophobic system $\varepsilon_{c o}^{h}$ are on the right-hand side (b) and (d).

distance. To estimate $d_{0}$, we first compute the density profile from the center of the structure (whether parallel walls or nanotube) to the wall. Then, we calculate the distance between the $Z$ value (for planar confinements) or $r$ value (for cylindrical structures) at the highest density $(d(\rho=\max ))$ and $Z$ or $r$ position at zero density $(d(\rho=0)$, as shown in Fig. 2). Finally diving by two (for symmetry reasons), we compute $d_{0}$ as

$$
d_{0}=\frac{1}{2} \sigma_{C O}-\frac{1}{2}(|d(\rho=\max )-d(\rho=0)|)
$$

The effective distance $\left(d_{e f f}\right)$, volume $\left(V_{e f f}\right)$ and density $\left(\rho_{e f f}\right)$, for all the systems considered in this work are reported in Table III.

Figure 3 represents the density profile obtained for con- 
fined water under planar confinement (W-1 and W-2) in a wide temperature range, considering the hydrophobic and superhydrophobic walls. The top panels of Fig. 3 are the results for the W-1 superhydrophobic (Fig.3-a) and hydrophobic (Fig.3-b) slab geometries. In the superhydrophobic case, the first peaks are more separated from the walls than in the hydrophobic one. A similar behavior has been observed in Ref. [36, 49], where the authors studied a confinement between parallel walls at a similar separation $(1.7 \mathrm{~nm})$. In the hydrophobic case, due to the stronger water-wall interaction, the first peaks appear closer to the walls, reaching a maximum density of $\rho=2.25 \mathrm{~g} / \mathrm{cm}^{3}$, almost twice the maximum value reached for $\varepsilon_{c o}^{s h}$. This result implies that water is more structured close to the wall in the hydrophobic system than in the superhydrophobic one.

A similar effect is observed for the W-2 superhydrophobic case (see Fig. 3) As expected, in the middle of the simulation box, where the effect of the interface disappears, the system behaves as in the bulk phase and the density profile resembles the bulk density value independently on the $\varepsilon_{C O}$ value used.

Figure 4 presents the density profiles obtained for water confined in CNT-1, CNT-2 and CNT-3 nanotubes in a wide temperature range, considering hydrophobic and superhydrophobic walls. When the interaction is superhydrophobic, changing the geometry of the confinement (from planar walls to cylinders) does not affect what was already observed for planar walls: the peaks of the density profile are farther away from the surface than in the hydrophobic case while the peaks heights are higher in the latter case. Focusing on the CNT-1 system, when the interactions with the surface are hydrophobic (Fig. 4-b), the water density increases as approaching to the CNT surface reaching a density maximum of $\rho=1.23 \mathrm{~g} / \mathrm{cm}^{3}$ at $r=0.90 \mathrm{~nm}$ at the highest temperature $(T=298 \mathrm{~K})$. When considering superhydrophobic interactions (Fig. 4a), this peak is lower $\left(\rho=1.14 \mathrm{~g} / \mathrm{cm}^{3}\right.$ at $\left.r=0.85 \mathrm{~nm}\right)$. Interestingly, the highest peak of the CNT-1's density profile, the one with the largest curvature, presents a shoulder that is closer to the carbon atoms than water confined in any other other system. This shoulder, originated by the curvature inducing a closer interaction between water and carbon, is the signature that the large curvature forces water to structure close to the hydrophobic nanotube.

Similarly, in the CNT-2 a similar displacement of the maximum of the density profile can be detected: the maximum in the density profile reaches a value of $\rho=1.26 \mathrm{~g} / \mathrm{cm}^{3}$ at $r=2.03 \mathrm{~nm}$ in the hydrophobic case (Fig. 4-d) and of $\rho=1.03 \mathrm{~g} / \mathrm{cm}^{3}$ at $r=1.86 \mathrm{~nm}$ in the superhydrophobic case (Fig. 4-c). Moreover, in the latter case, the density profile slightly decreases with increasing temperature. Also in the CNT-3 a similar displacement of the maximum of the density profile can be detected: the density reaches a maximum at $\rho=1.24 \mathrm{~g} / \mathrm{cm}^{3}$ and fluctuates around this value independently of the water-
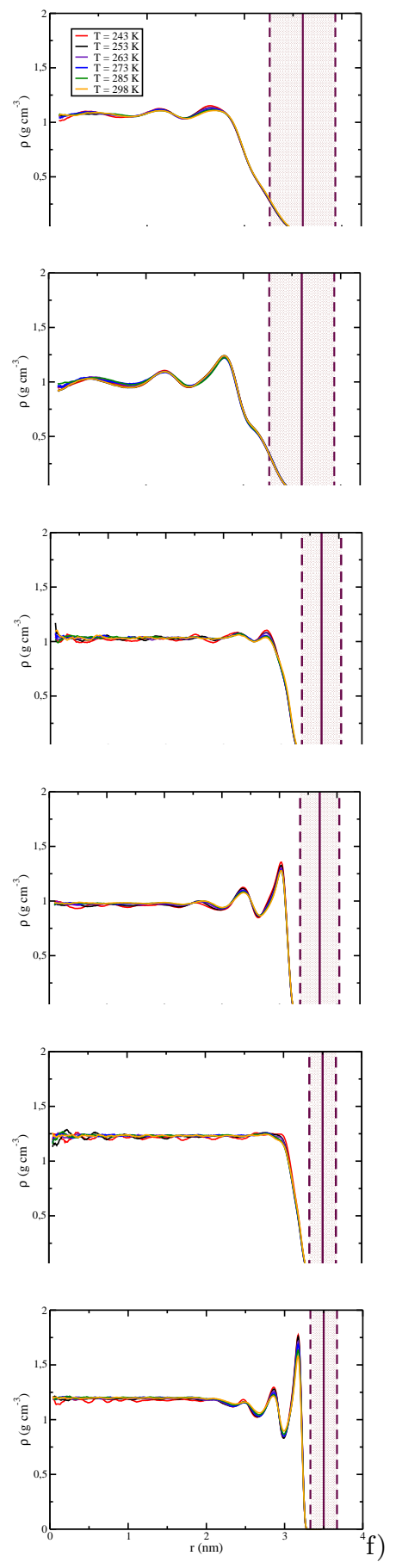

FIG. 4: Density profiles for water confined in CNT-1 (top panels), CNT-2 (middle panels) and CNT-3 (bottom panels) geometries at different temperatures (legend shown in figure a)). Results for the superhydrophobic system $\varepsilon_{c o}^{s h}$ are on the left-hand side ( $\mathrm{a}, \mathrm{c}$ and e) whereas for the hydrophobic system are on the right-hand side $\varepsilon_{c o}^{h}(\mathrm{~b}, \mathrm{~d}$ and $\mathrm{f})$. 

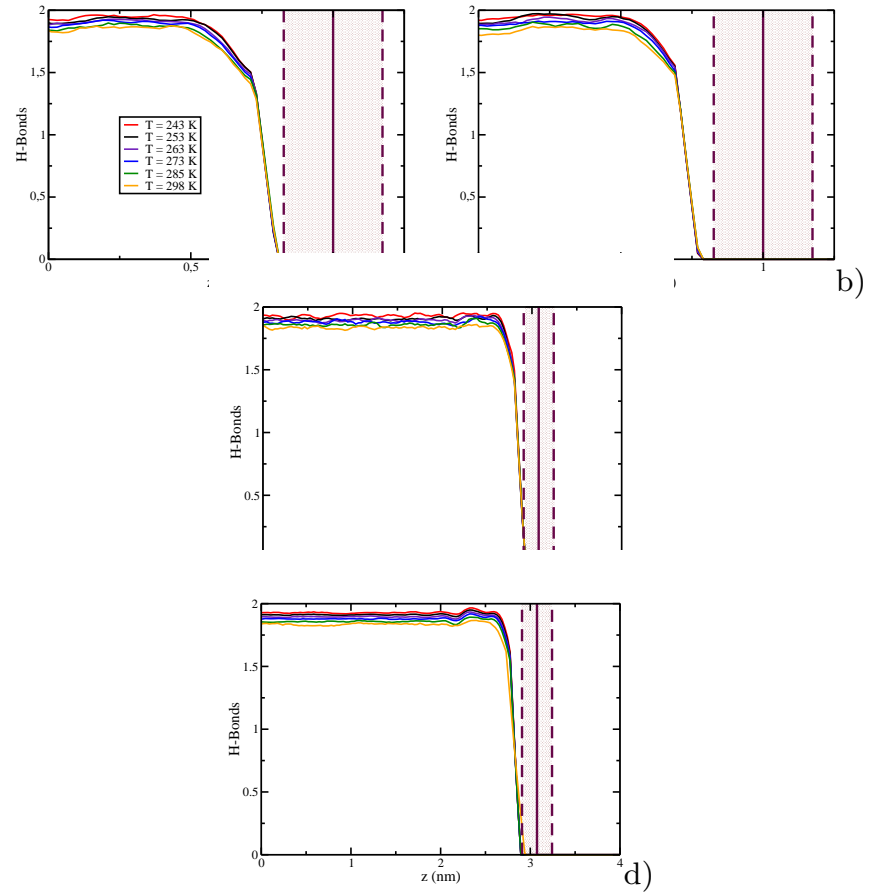

FIG. 5: Number of hydrogen bonds per molecule calculated for water confined between parallel walls: W-1 (top panels) and W-2 (bottom panels). Left-hand side (a and c) superhydrophobic and right-hand side (b and d) hydrophobic surfaces.

carbon interaction (see Fig. 4-e and Fig. 4-f).

As can be observed for all cases considered in Figs. 4 and 2 , the effect of the temperature on the density profiles is not very noticeable.

As expected, the density profiles obtained for CNT-1 and CNT-2 are in excellent agreement with the results reported by Köhler et al. [48] for CNT $(16,16)$ (at $\rho_{1}=$ $0.93 \mathrm{~g} / \mathrm{cm}^{3}$ and $\rho_{2}=1.12 \mathrm{~g} / \mathrm{cm}^{3}$ ) and for CNT $(30,30)$ (at $\rho_{3}=0.95 \mathrm{~g} / \mathrm{cm}^{3}$ and $\rho_{4}=1.15 \mathrm{~g} / \mathrm{cm}^{3}$ ) using TIP $4 \mathrm{P} / 2005$ as water model.

\section{B. Hydrogen bonds profiles}

To further study the molecular structure of confined water, we analyzed how the average number of HBs of water molecules varies with respect to the distance from the confining surface.

A water molecule can be donor and acceptor of two hydrogen bonds. In such way, we chose one of the two possible roles of each molecule and we computed the HBs average using this criterion: therefore the average number of $\mathrm{HB}$ of a bulk molecule is around 2 .

When water is confined, the number of HBs per molecule decreases, especially close to the (super)hydrophobic surface. Figure 5 shows the average number of hydrogen bonds for confined water between two parallel walls ( $\mathrm{a}$ and $\mathrm{b}$ for $\mathrm{W}-1-$ and $\mathrm{c}$ and $\mathrm{d}$ for $\mathrm{W}$ -
2) within a wide temperature range. Similarly, Fig. 6 represents the analogous property for a cylindrical confinement ( $a$ and $b$ for CNT-1, $c$ and d for CNT-2 and e and $\mathrm{f}$ for CNT-2). As in bulk water, in every system in Fig. 5 and Fig. 6, we observe that the number of HBs decreases as the temperature increases, given that a higher temperature corresponds to a higher kinetic energy that leads to a higher bond breakage.

Close to the wall, regardless the type of confinement, the number of HBs decreases until zero showing a region with a value less than 2 , in good agreement with the Hydrogen bonds profile studied previously by Werder and coworkers. [100] Due to the structuring of liquid water in the proximities of the surface (as shown in Figs. 3 and 4), more pronounced in the hydrophobic than in the superhydrophobic system, the number of HBs presented is on average higher closer to the surface, especially for the hydrophobic systems.

Focusing on CNT-1, we observe a shoulder (especially in the hydrophobic case) on the number of HBs near the surface together with a non-zero number of HBs inside the surface: these are both effects of large curvature of the system. However, for both CNT-2 and CNT-3 systems the number of HBs is always around 2, being slightly higher before approaching the surface. At that point the number of HBs dramatically drops to zero, due to the (super)hydrophobicity of the walls. In the CNT-2 and CNT-3 geometries, curvature effects are almost negligible while the bulk contribution (towards the center of the nanotubes) increases.

\section{Diffusion Coefficient}

Having studied the structural properties of water under nano-confinements, we computed its transport properties for planar confinements (W-1 and $\mathrm{W}-2$ ) and for two cylindrical confinements (CNT-1 and CNT-2). In the latter case, we are not considering CNT-3 since this system behaves as the bulk. Therefore the CNT-2 system is enough to draw conclusions about nanotubes with a large diameter.

We first compute the diffusion coefficient $D$ in a wide temperature range $(243 \mathrm{~K}-298 \mathrm{~K})$ and for the different hydrophobicities of the surface.

As discussed in section II, the effect of the finite lateral size of the simulation box is simply dealt with by computing the mean square displacement of the particles after removing the motion of the center of mass; in confined systems, this provides a reasonable estimate of the intrinsic self-diffusion coefficient (i.e., in the limit of an infinite lateral box size). Therefore, the diffusion coefficients presented are the bare values measured from the mean square displacement.

Results for water in planar confinement (W-1 and W2 and the two different hydrophobicities) are presented 

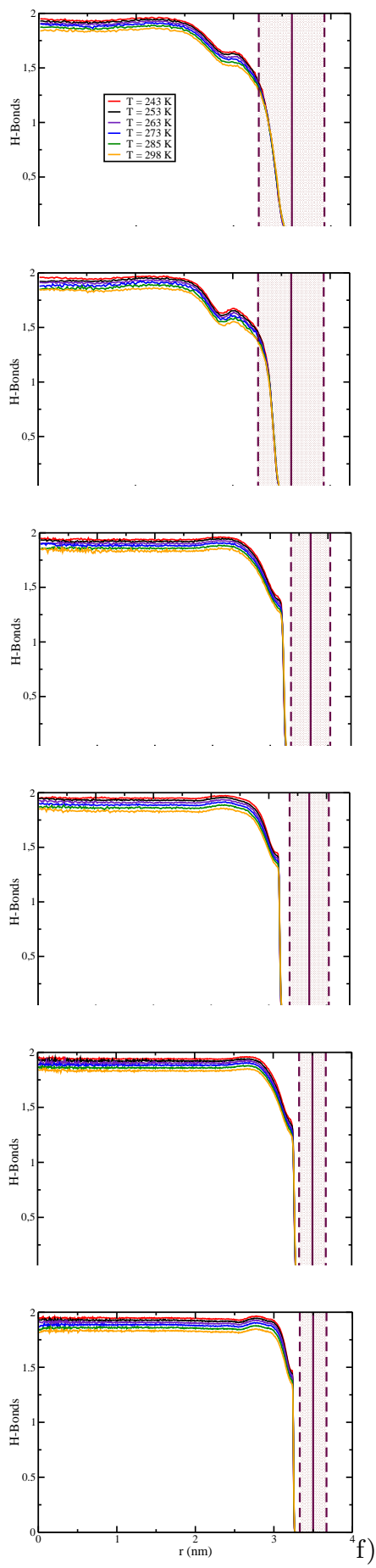

FIG. 6: Number of hydrogen bonds per molecule calculated for water confined inside nanotubes: CNT-1 (top panels), CNT-2 (middle panels) and CNT-3 (bottom panels). Lefthand side ( $\mathrm{a}, \mathrm{c}$ and $\mathrm{e}$ ) superhydrophobic and right-hand side (b, d and f) hydrophobic surfaces.
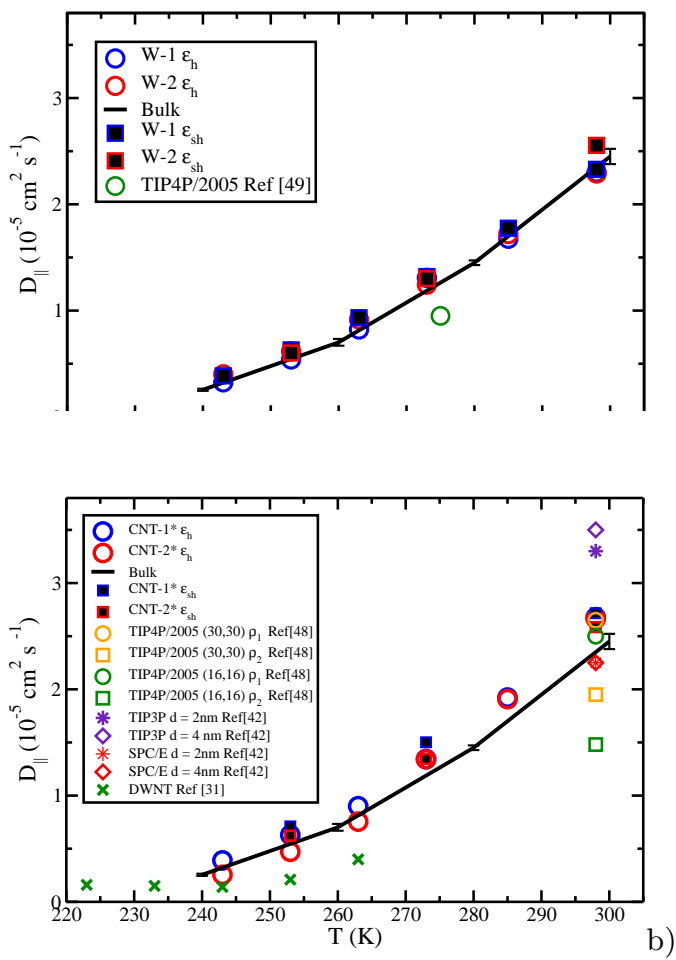

FIG. 7: Diffusion coefficient of water as a function of temperature when confined a) between hydrophobic and superhydrophobic walls, b) inside hydrophobic and superhydrophobic nanotubes. The green empty circle in a) is for water confined between sheets $(\mathrm{d}=1.4 \mathrm{~nm})$ from Ref. [49]. Bulk diffusion coefficient from Ref. [98] is represented by a black line. Stars and diamonds in panel $\mathrm{b}$ represent the diffusion inside nanotubes with a diameter of 2-4 $\mathrm{nm}$ for TIP3P water (purple) and SPC/E water (red) from Ref. [42]. Orange and green symbols represent results for water at densities $\rho=0.95 \mathrm{~g} / \mathrm{cm}^{3}$ and $\rho=1.15 \mathrm{~g} / \mathrm{cm}^{3}$ (circles and squares respectively) nanotubes with a chirality of $(16,16)$ and $(30,30)$ from Ref. [48].

in Fig. 7-a, which shows that the diffusion coefficient of water confined under parallel walls is independent of the distance between walls (W-1 or W-2), and is similar to that of bulk water in the same temperature range: at low temperatures, the system presents slower diffusion. Moreover, the amount of hydrophobicity of the surface does not affect significantly the diffusion coefficient. In the same panel we also report the result from Ref. [49] obtained for TIP $4 \mathrm{P} / 2005$ water in a more stringent confinement. The discrepancy with respect to our results might be due to the fact that water in Ref. [49] is confined in a much tighter slab. This leads to an even more pronounced structuring of liquid water, that strongly affects its dynamic properties.

The results obtained for CNTs are presented in Fig. 7b. Interestingly, the diffusion coefficient is affected by the curvature, being higher for the most curved surfaces (CNT-1 and CNT-2), and is barely affected by the hydrophobicity of the surface (being slightly higher in the 
hydrophobic system). As in the parallel walls confinements, the behavior of the diffusion coefficient with temperature is similar to the bulk. When $T \leq 263 \mathrm{~K}$, the values of the diffusion coefficient resemble those of bulk water independently on the curvature of the geometries and on their hydrophobicity. In contrast, at high temperature, the diffusion coefficient of confined water is higher than the corresponding value for bulk water.

Focusing on $T=298 \mathrm{~K}$, where the effect is more dramatic, we compared our results with results from the literature. As already suggested in Ref. [58], the diffusion coefficient of confined water is strongly affected by the water model,with TIP3P giving values too high with respect to SPC/E.[42] When comparing with TIP4P/2005 confined water at similar density and for similar chirality (orange symbols), we obtain results in perfect agreement with the literaturei. [48]

Therefore, water diffuses faster when confined in hydrophobic nanotubes with an effective diameter ranging from $2 \mathrm{~nm}$ up to about $4 \mathrm{~nm}$ (CNT-1 and CNT-2, respectively).

In order to unravel the microscopic origin of this behavior, we evaluate how the diffusion coefficient varies with the distance from the hydrophobic surface.

Figure 8 represents the diffusion coefficient of water molecules when confined: a) between parallel walls (W-1) at $298 \mathrm{~K}$ and inside an hydrophobic nanotube (CNT-1) at $298 \mathrm{~K}$ (b) and at $273 \mathrm{~K}$ (c). Densities of water confined in those systems are similar, but curvatures and structures are definitively different. Data for $D$ are plotted along the $X Y$ direction for $\mathrm{W}-1$ (Fig. 8-a) and the $Z$ direction for CNT-1 (Fig. 8-b and 8-c).

For the slit pore at $298 \mathrm{~K}$ (W-1, Fig. 8-a), we observe that the average diffusion, computed with Eq. (2) (green line) is practically the same as that for bulk water (continuous black line). Figure 8-a also shows the prediction of continuum hydrodynamics for the local diffusivity, Eq. (B2). The shape of the theoretical curve matches well the measured values, except very close to the walls, where the diffusivity increment is lower than the continuum prediction. On the one hand, this could simply be because Eq. (B2) was derived for a distance to the walls $z \gg \sigma_{\mathrm{h}}$, so that it would be outside its range of validity. On the other hand, the large quantitative discrepancy could be due to beyond-continuum effects, related in particular to the large changes in the (H-bond) structure highlighted in sections 3.1 and 3.2.

Note that in Eq. (B2), the confined Stokes-Einstein viscosity, $\eta_{\mathrm{SE}}$ was used, so that the average of Eq. (B2) corresponds to $\left\langle D_{\|}\right\rangle$. As a consequence, because $D_{\|}(z)$ is overestimated by Eq. (B2) close to the walls, it is also slightly underestimated in the middle of the slab, by ca. $3 \%$. This value provides an estimate of the typical error in the viscosity computed by the confined StokesEinstein method, where Eq. (B2) is assumed to be valid everywhere in the slab.

As shown in Fig. 8-b and Fig. 8-c, the curvature plays
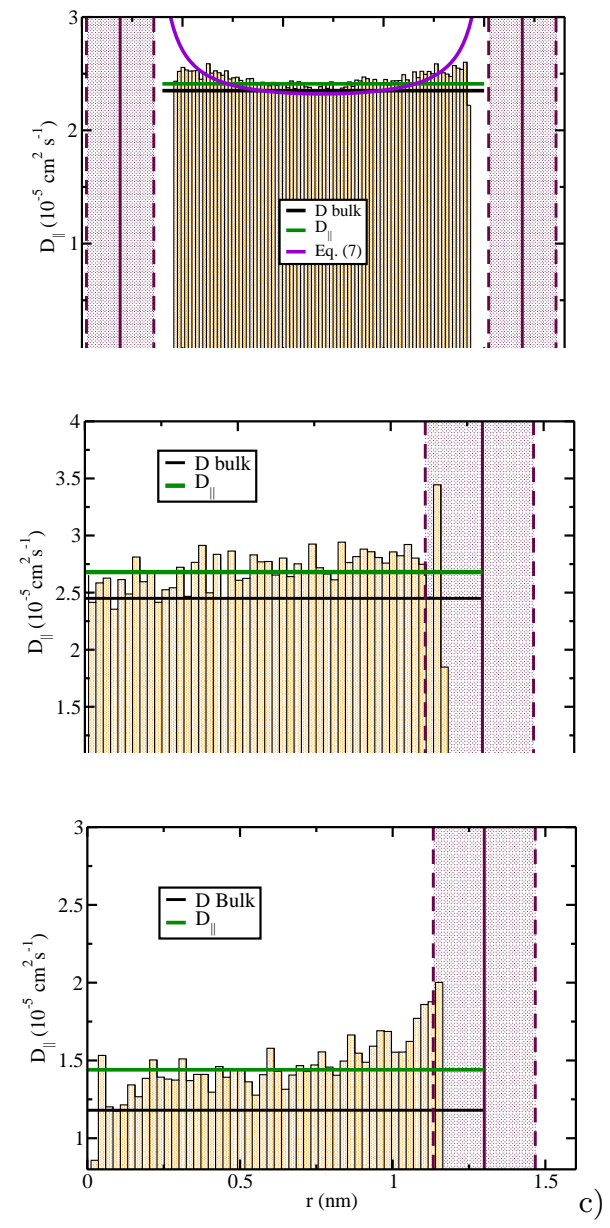

FIG. 8: Diffusion coefficient along the distance between walls or the radius of the nanotube for $\mathrm{W}-1$ at $T=298 \mathrm{~K}$ (panel a), CNT- 1 at $T=298 \mathrm{~K}$ (panel b) and at $T=273 \mathrm{~K}$ (panel c). The green line represents the diffusion value reported in Fig. 7 at the same conditions and the black line is the diffusion of bulk water from Ref. [98].The purple line represents the diffusivity profile calculated via Eq. (B2).

an important role in determining the diffusion of water, as previously suggested. [22, 101] When considering the effect of the curvature (CNT-1) we observe that the average diffusion is higher than the value for bulk water at 298 K (Fig. 8-a), and $273 \mathrm{~K}$ (Fig. 8-c).

\section{Viscosity}

We now focus on the calculation of the viscosity, which we evaluate via two routes: the Green-Kubo expression, given in Eq. (1) and the confined Stokes-Einstein expression, given by Eq. (4), derived using continuum hydrodynamics. Concerning the Green-Kubo approach, as explained in Sec. II, we use only some components of the pressure tensor, depending on the geometry of the confinement. Therefore, care must be taken when compar- 

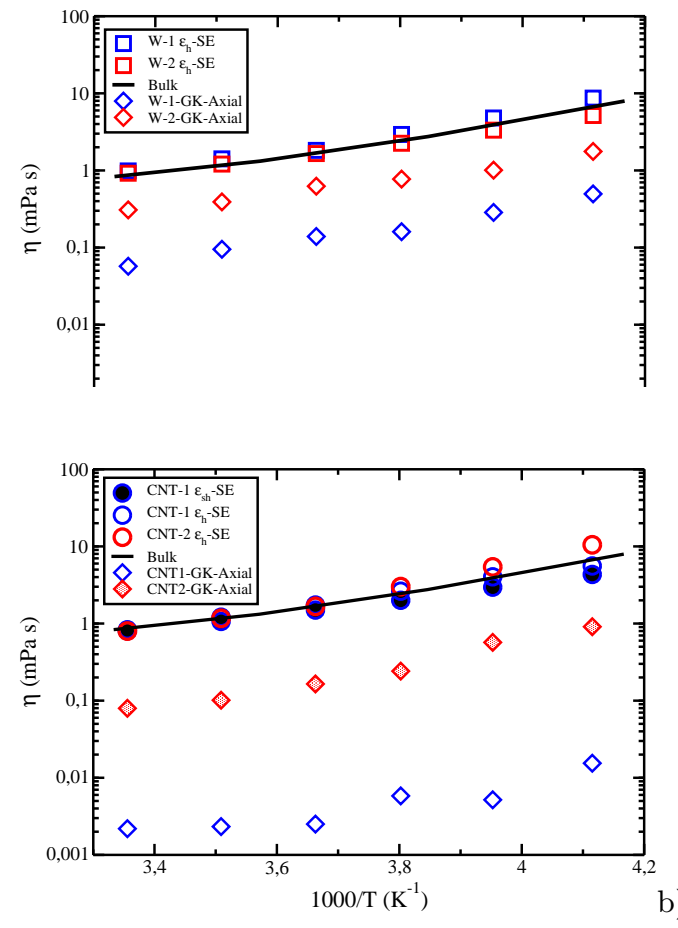

FIG. 9: Viscosity $\eta$ as a function of temperature for water in slits (a) and in nanotubes (b). Blue color represents the smallest system in each case (W-1 or CNT-1) and red color represents W-2 or CNT-2. Black line shows the results reported in Ref.[98] for bulk. The Green-Kubo estimate (diamonds) and the confined Stokes-Einstein estimate (squares and circles) are compared.

ing to literature results obtained for bulk water, where all pressure tensor's components are taken into account.

We focused on the hydrophobic case, computing the viscosity for both slit pore and nanotube systems in a wide temperature range (243-298 K). Our results are presented in Fig. 9-a for the hydrophobic slits and Fig. 9$\mathrm{b}$ for CNT-1 and CNT-2 nanotubes. Consistently with the diffusivity, the confined Stokes-Einstein viscosity reported in panel a for a slit pore shows a very good agreement with the bulk values (continuous black line) independently on the system size (W-1 blue and W-2 red squares). In contrast, the Green-Kubo axial viscosity (empty diamonds) depends strongly on system size (with the largest W-2 system closer to the bulk values than the $\mathrm{W}-1$ one). Note that similar results for the viscosity have been obtained using the GK expression by ref. [48] for a similar size CNT (same density and hydrophobicity).

Similar results are obtained for nanotubes (panel b): $\eta_{\mathrm{SE}}$ is always very close to the bulk values (continuous black line) independently of the system size (CNT-1 blue and CNT-2 red circles) and on hydrophobicity ( $\varepsilon_{c o}^{h}$ empty and $\varepsilon_{c o}^{s h}$ filled symbols). A small discrepancy appears at low temperatures, which could be related to the failure of the Stokes-Einstein relation in supercooled water. In contrast, a clear system size dependence is detected for the Green-Kubo axial viscosity (empty diamonds); for the largest CNT-2 system viscosity values are closer to those of the bulk when compared with those of the CNT1 one.

To conclude, while viscosity computed with the GreenKubo formula (applied for anisotropic and confined system) strongly differs from the bulk, viscosity computed with the confined Stokes-Einstein relation is not so affected by confinement, independently on its geometry.

\section{SUMMARY AND CONCLUSIONS}

In this work we present a thorough study of TIP4P/2005 water confined in different hydrophobic nanostructures within a wide temperature range. Properties such as density profiles, HBs per molecule, diffusion and viscosity were obtained using MD simulation.

Studying the density profiles, we concluded that water density approaches the bulk value in the middle of either the slit pore or the nanotube, while more structured water (highest peaks) is observed near the walls. We detect differences of the density profiles depending on the amount of hydrophobicity considered: water seems more structured in the hydrophobic cases rather than in the superhydrophobic ones. As expected, the number of HBs in bulk water is reached in the middle of either the slit pore or the nanotube; while near the surface a sharp decrease to zero is observed, given that those molecules have a smaller number of water neighbours, thus forming less bonds. Interestingly, in the CNT-1 system, we observe a shoulder that we attribute to curvature effects.

To study diffusivity, we treated effects of the finite lateral size of the simulation box by simply computing the mean squared displacement of the molecules after removing the motion of the liquid center of mass. Interestingly, when confined in nanometer size nanotubes, water tends to diffuse faster than in bulk (CNT-1 and CNT-2). Considering that CNT-1 has the highest curvature, we conclude that curvature is an important parameter that can be handled to produce an increment of the diffusion while confining water, especially at lower temperatures. We showed that the confinement of water induces a significant difference between the diffusion of the molecules in the center of the systems and the molecules close to the interface as shown in figure 8 , in qualitative agreement with the prediction of continuum hydrodynamics. The quantitative discrepancy could be either due to an approximation in the derivation of the analytical prediction, or to non-continuum effects, consistently with the large structural changes close to the walls.

We then compared the two methods to evaluate viscosity. First, we used a Green-Kubo expression (whose applicability in confined heterogeneous systems is not guaranteed), considering only some components of the pres- 
sure tensor for computing axial viscosity as commented in Sec. 2.3. Then, we derived a confined Stokes-Einstein relation, taking into account the influence of the (stressfree) confining walls, based on a previous expression derived for no-slip walls [85]. Viscosity can also be estimated through this confined Stokes-Einstein relation, although this expression could fail at low temperatures, similarly to its bulk counterpart. We found that the two estimates differ dramatically, with the confined StokesEinstein estimate globally remaining closer to the bulk viscosity, and being more consistent with what we observed for diffusion (by construction). Since both methods have their shortcomings, here we only would like to suggest that measuring viscosity in a confined system is a delicate business, and that different methods may provide dramatically different results, which may not be easily related to the standard, experimental definition of viscosity. To conclude, care must be taken when computing viscosity in inhomogeneous and anisotropic confined systems. In terms of diffusion and viscosity, we do not see any dynamical signature of any liquid-liquid transition. At least within the chosen temperature range and nanotube diameter/plane geometry. Further work is needed to unravel the features of water under ultra-confinement, where water could undergo structural and/or dynamical transitions. [102-105]

\section{Acknowledgements}

A.Z. thanks CONACYT for the $\mathrm{PhD}$ scholarship. M.A.G. acknowledges support by Ayuda Juan de la Cierva-Incorporacin (IJCI-2016-27497) from Ministerio de Ciencia, innovación y universidades (Spain). This work was partially supported by CNRS (France) through a PICS program. L.J. acknowledges support from Institut Universitaire de France. C.V. acknowledges funding from Grant FIS2016-78847-P of the MINECO and together with I.L.M. and M.A.C.M. the UCM/Santander PR26/16-10B-2. The authors acknowledge the computer resources and technical assistance provided by Marenostrum (RES).

\section{Appendix A: Contact angle measurements}

In order to quantify the amount of hydrophobicity, we compute the contact angle of a liquid droplet located on a planar surface. As in an experimental drop-shape analysis [106], we prepare a liquid droplet containing 200 water molecules and locate it on top of a rigid and flat carbon surface. Depending on whether the surface is hydrophobic (h) or superhydrophobic (sh), the surface will be "partially wet" as we can see in Fig. 10-a or dry as in Fig. 10-b.

Next, we estimated the contact angle between the

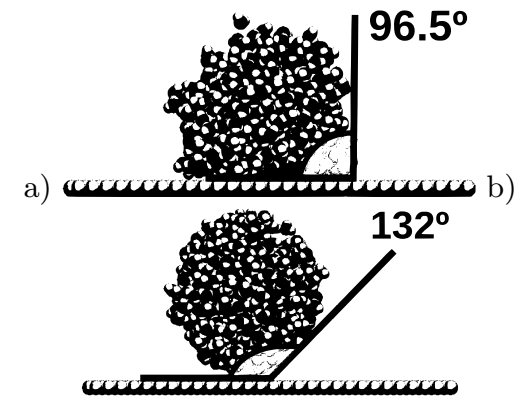

FIG. 10: Snapshots representing liquid droplets on a flat surface. Carbon atoms are represented by cyan spheres and the water molecules are red and white (red for oxygen and white for hydrogen). (a) Hydrophobic water-wall interaction using $\varepsilon_{c o}^{h}$ (b) Superhydrophobic water-wall interaction using $\varepsilon_{c o}^{s h}$. The numbers indicate the contact angles resulting from our calculations.

droplet and the surface. After having equilibrated the system for $2 \mathrm{~ns}$, we measured the contact angle by projecting on the $X-Y$ plane the water molecules and of the carbon atoms coordinates: defining the line formed by the outermost molecules in the droplet with the molecule in contact with the layer, and a line defined on the plane of the carbon atoms, then we computed the contact angle among them. To get more statistics, we have used the coordinates of all molecules for 10 independent trajectories at same temperature, averaging the final result.

With this method, we estimated the contact angle for the hydrophobic case to be around $96.5^{\circ}$ which indicates a slight hydrophobicity of the surface, similarly to the one computed by Hummer et al.[21], Moskowitz et al.,[71] and Kohler et al.[48] considering the same interaction strength $(\varepsilon=0.2703 \mathrm{~kJ} / \mathrm{mol})$. In the superhydrophobic system, where $\varepsilon_{c o}^{s h}$ is $0.047 \mathrm{~kJ} / \mathrm{mol}$, we obtained a contact angle of $\approx 132^{\circ}$, much larger than $90^{\circ}$.

\section{Appendix B: Confined Stokes-Einstein relation}

Here we consider a liquid confined in an infinite slit pore of height $d$, assuming a perfect slip (stress-free) boundary condition at the walls, and a homogeneous and isotropic viscosity $\eta$. We derive an expression for the average self-diffusion coefficient parallel to the walls. To that aim, we follow the approach described in Ref. 85, with the only difference that we consider stress-free walls.

We define the vertical position $z$ by the position of the hydrodynamic shear planes at $z=0$ and $d$. The local diffusivity $D_{\|}(z)$ can be related to the local mobility $\mu_{\|}(z)$ through Einstein relation [92]: $D_{\|}(z)=k_{\mathrm{B}} T \mu_{\|}(z)$. The mobility can then be computed within the framework of continuum hydrodynamics. In bulk, the standard Stokes prediction $1 / \mu_{\|}^{\text {bulk }}=3 \pi \eta \sigma_{\mathrm{h}}$ can be used to define the effective hydrodynamic diameter of the particles $\sigma_{\mathrm{h}}$, see Table IV. In confinement, the mobility is modified due to 
TABLE IV: Bulk diffusivity $D_{\text {bulk }}$, bulk viscosity $\eta_{\text {bulk }}$ and effective hydrodynamic diameter $\sigma_{\mathrm{h}}=k_{\mathrm{B}} T /\left(3 \pi \eta_{\mathrm{bulk}} D_{\mathrm{bulk}}\right)$ as a function of temperature for a density of $999.26 \mathrm{~kg} / \mathrm{m}^{3}$. Data extrapolated from Ref. 98 (we fitted the numerical data with Vogel-Tammann-Fulcher laws).

\begin{tabular}{|c|c|c|c|}
\hline \hline$T(\mathrm{~K})$ & $D_{\text {bulk }}\left(\times 10^{-9} \mathrm{~m}^{2} / \mathrm{s}\right)$ & $\eta_{\text {bulk }}(\mathrm{mPas})$ & $\sigma_{\mathrm{h}}(\mathrm{nm})$ \\
\hline 298 & 2.35 & 0.849 & 0.219 \\
285 & 1.67 & 1.18 & 0.212 \\
273 & 1.14 & 1.71 & 0.205 \\
263 & 0.791 & 2.47 & 0.197 \\
253 & 0.513 & 3.81 & 0.190 \\
243 & 0.306 & 6.44 & 0.181 \\
\hline \hline
\end{tabular}

friction on the two walls. When the distance to the walls is large as compared to the particle radius, one can write [85, 87-89]:

$$
\frac{1}{\mu_{\|}(z)}=3 \pi \eta \sigma_{\mathrm{h}}\left(\frac{1}{1+\frac{3}{8} \frac{\sigma_{\mathrm{h}}}{2 z+\sigma_{\mathrm{h}}}}+\frac{1}{1+\frac{3}{8} \frac{\sigma_{\mathrm{h}}}{2(d-z)+\sigma_{\mathrm{h}}}}-1\right) .
$$

The local diffusivity is accordingly:

$D_{\|}(z)=\frac{k_{\mathrm{B}} T}{3 \pi \eta \sigma_{\mathrm{h}}}\left(\frac{1}{1+\frac{3}{8} \frac{\sigma_{\mathrm{h}}}{2 z+\sigma_{\mathrm{h}}}}+\frac{1}{1+\frac{3}{8} \frac{\sigma_{\mathrm{h}}}{2(d-z)+\sigma_{\mathrm{h}}}}-1\right)^{-1}$.

The average diffusivity is then given by:

$$
\left\langle D_{\|}\right\rangle=\frac{1}{d} \int_{0}^{d} D_{\|}(z) \mathrm{d} z
$$

The full solution is rather cumbersome, but in large pores $\left(d \gg \sigma_{\mathrm{h}}\right)$, it can be approximated by:

$$
\left\langle D_{\|}\right\rangle \approx \frac{k_{\mathrm{B}} T}{3 \pi \eta \sigma_{\mathrm{h}}}\left[1+\frac{3}{8} \frac{\sigma_{\mathrm{h}}}{d} \ln \left(\frac{2 d}{\sigma_{\mathrm{h}}}\right)\right] .
$$

In the main text, we will refer to this expression as the confined Stokes-Einstein relation, and use it to compute the liquid viscosity from its diffusion coefficient. In practice, we will use the effective wall position as the origin, and the effective wall distance for $d$ (see section 3.1 of the main text).

\section{Appendix C: Experimental NMR measurements}

In order to confirm our numerical results, we have attempted to measure the diffusion coefficient inside the nanotubes by means of Nuclear Magnetic Resonance, NMR, following the protocol described in Ref. [31], that uses NMR DOSY (Diffussion Ordered SpectroscopY) experiments to experimentally determined the diffusion coefficients.

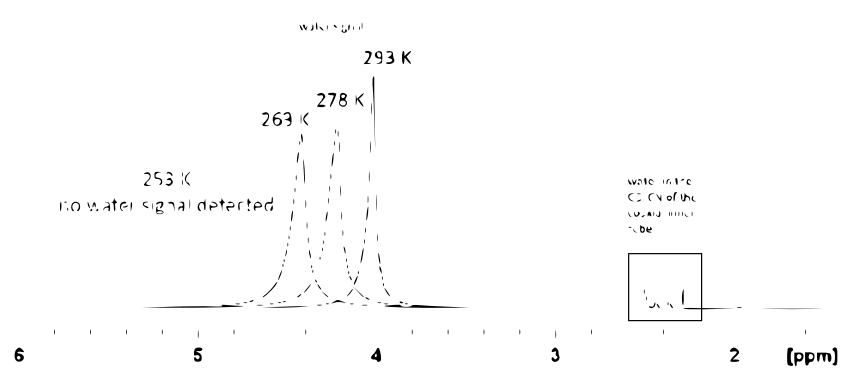

FIG. 11: ${ }^{1} \mathrm{H}$ spectra of the CNTs-water suspension at $293 \mathrm{~K}$ (black curve), $278 \mathrm{~K}$ (red curve), $263 \mathrm{~K}$ (dark blue) and $253 \mathrm{~K}$ (green).

To start with, we prepare a suspension of single walled CNTs in water. We located a suspension of $300 \mu \mathrm{l}$ distilled water and $1 \mathrm{mg}$ single walled CNTs with open tips at both ends (provided by Ionic liquid Technologies, OSWCNT diameter $=1-2 \mathrm{~nm}$, length $=1-3 \mu \mathrm{m}$ ) in a 5 $\mathrm{mm}$ NMR tube. To properly disperse the nanotubes in water, we sonicate the suspension for 6 hours.

Next, we have inserted in the sample a coaxial inner tube containing CD3CN needed as an external lock solvent for the NMR experiment. We acquired ${ }^{1} \mathrm{H} 1 \mathrm{D}$ NMR experiments on a Bruker Advance $500 \mathrm{MHz}$ spectrometer with 16 scans at the following temperatures: $293 \mathrm{~K}, 278 \mathrm{~K}, 263 \mathrm{~K}$ and $253 \mathrm{~K}$. Next, we performed NMR DOSY (Diffusion Ordered SpectroscopY) experiments at $500 \mathrm{MHz}$ with the standard Bruker DOSY protocol (stebpgp1s), collecting thirty two $1 \mathrm{D}{ }^{1} \mathrm{H}$ spectra with a gradient duration of $\delta=1 \mathrm{~ms}$ and an echo delay of $\Delta=100 \mathrm{~ms}$.

NMR spectra are shown in Fig. 11. As expected, the water signal shifts to the left for lower temperatures.

The ${ }^{1} \mathrm{H}$ NMR signal at $263 \mathrm{~K}$ displays line broadening (as its signal in the DOSY experiment), signature of the fact that the sample contains a mixture of bulk and frozen water $\left(\log \left(K_{D}\right)=-(9.02-9.14)\right.$ thus $\left.K_{D}=\left(9.5 * 10^{-10}-7.2 * 10^{-10}\right) \mathrm{m}^{2} / \mathrm{s}\right)$. No signal has been detected at the lowest temperature of $253 \mathrm{~K}$ due to the crystallization of the entire suspension. Interestingly, at every temperature we always noticed on the right a low peak that we attribute to a small amount of water present in the CD3CN capillary.

Results obtained from the NMR DOSY experiments are shown in Fig.12.

As reported in Fig 11, the suspension is liquid at temperatures down to $263 \mathrm{~K}$. As shown in Table V, the obtained values of the diffusion coefficient at $298 \mathrm{~K}, 293 \mathrm{~K}$ and $278 \mathrm{~K}\left(\log \left(K_{D}\right)=-8.59,-8.64,-8.85\right.$, respectively $)$ are consistent with the reported values for bulk water presented in the literature.

Our experimental results for bulk water are represented together with our numerical results for water confined in the hydrophobic $C N T 1$ and $C N T 2$ nanotube 


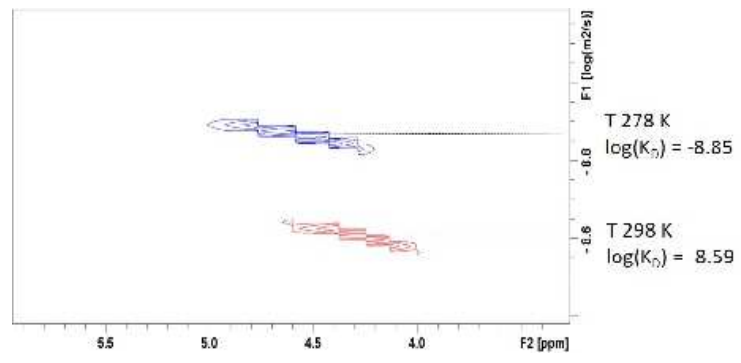

FIG. 12: DOSY NMR experiments acquired at $298 \mathrm{~K}$ (red colour) and $278 \mathrm{~K}$ (blue colour) overlaid, where $\log \left(K_{D}\right)=$ $-8.85($ at $278 \mathrm{~K})$ and $\log \left(K_{D}\right)=-8.59($ at $298 \mathrm{~K})$.

\begin{tabular}{|c|c|c|}
\hline \hline $\mathrm{T}(\mathrm{K})$ & $\mathrm{D}\left(\mathrm{cm}^{2} \mathrm{~s}^{-1}\right)$ & $\mathrm{D}\left(\mathrm{cm}^{2} \mathrm{~s}^{-1}\right)$ Literature \\
\hline \hline 298 & $2.57 \times 10^{-5}$ & $2.29 \times 10^{-5}$ Ref.[? ] \\
\hline 293 & $2.29 \times 10^{-5}$ & $2.02 \times 10^{-5}$ Ref.[? ] \\
\hline 278 & $1.41 \times 10^{-5}$ & $1.31 \times 10^{-5}$ Ref.[? ] \\
\hline \hline
\end{tabular}

TABLE V: Comparison between experimental and reported bulk water diffusion coefficients

and with the results presented in reference [31].

Differently from us, Liu et al. [31] detected the ${ }^{1} \mathrm{H}$ water NMR signal at temperatures below $263 \mathrm{~K}$ : the authors attributed this signal to endohedral mobile water inside the nanotubes. One possible difference between the two experiments is the amount of confined water, that could not be enough for NMR detection in our experimental conditions. Even though the diameter of the nanotubes is similar in both experiments (1-2 nm vs $2.3 \mathrm{~nm}$ in [31]) their length is smaller in our experiments $(1-3 \mu \mathrm{m}$ vs $12-18 \mu \mathrm{m}$ in [31]). Another difference is that we used single wall, while the authors of [31] used multi walled nanotubes.

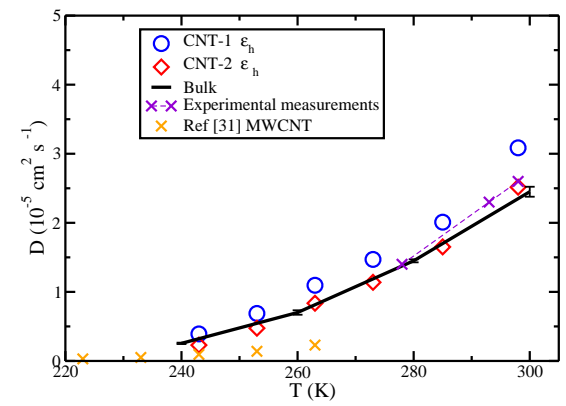

FIG. 13: Diffusion coefficient of water confined within $C N T 1$ and $C N T 2$ nanotubes (red and blue symbols). The purple symbols are results from our experiments while the orange ones from Ref. [31]. The solid black line represents the expected values for bulk water.
[1] Martin Chaplin. Water structure and science, 2006.

[2] Yutaka Maniwa, Hiromichi Kataura, Masatoshi Abe, Akiko Udaka, Shinzo Suzuki, Yohji Achiba, Hiroshi Kira, Kazuyuki Matsuda, Hiroaki Kadowaki, and Yutaka Okabe. Ordered water inside carbon nanotubes: formation of pentagonal to octagonal ice-nanotubes. Chemical Physics Letters, 401(4):534 - 538, 2005.

[3] Kenichiro Koga, GT Gao, Hideki Tanaka, and Xiao Cheng Zeng. Formation of ordered ice nanotubes inside carbon nanotubes. Nature, 412(6849):802, 2001.

[4] Daisuke Takaiwa, Itaru Hatano, Kenichiro Koga, and Hideki Tanaka. Phase diagram of water in carbon nanotubes. Proceedings of the National Academy of Sciences, 105(1):39-43, 2008.

[5] Kumar Varoon Agrawal, Steven Shimizu, Lee W. Drahushuk, Daniel Kilcoyne, and Michael S. Strano. Observation of extreme phase transition temperatures of water confined inside isolated carbon nanotubes. $\mathrm{Na}$ ture Nanotechnology, 12:267 EP -, Nov 2016. Article.

[6] Vitaly V. Chaban, Victor V. Prezhdo, and Oleg V. Prezhdo. Confinement by carbon nanotubes drastically alters the boiling and critical behavior of water droplets. ACS Nano, 6(3):2766-2773, 2012. PMID: 22352413.

[7] Mateus Henrique Köler and Leandro Barros da Silva. Size effects and the role of density on the viscosity of water confined in carbon nanotubes. Chemical Physics Letters, 645:38 - 41, 2016.
[8] Masanori Hashido, Akinori Kidera, and Mitsunori Ikeguchi. Water transport in aquaporins: Osmotic permeability matrix analysis of molecular dynamics simulations. Biophysical Journal, 93(2):373 - 385, 2007.

[9] Thierry O. Wambo, Roberto A. Rodriguez, and Liao Y. Chen. Computing osmotic permeabilities of aquaporins aqp4, aqp5, and glpf from near-equilibrium simulations. Biochimica et Biophysica Acta (BBA) - Biomembranes, 1859(8):1310 - 1316, 2017.

[10] Emad Tajkhorshid, Peter Nollert, Morten Ø. Jensen, Larry J. W. Miercke, Joseph O'Connell, Robert M. Stroud, and Klaus Schulten. Control of the selectivity of the aquaporin water channel family by global orientational tuning. Science, 296(5567):525-530, 2002.

[11] Yi Zhang, Yuhong Bai, and Bing Yan. Functionalized carbon nanotubes for potential medicinal applications. Drug Discovery Today, 15(11):428 - 435, 2010.

[12] Zixian Li, Andre Luis Branco de Barros, Daniel Cristian Ferreira Soares, Sara Nicole Moss, and Laleh Alisaraie. Functionalized single-walled carbon nanotubes: cellular uptake, biodistribution and applications in drug delivery. International Journal of Pharmaceutics, 524(1):41 - 54, 2017.

[13] Lingjie Meng, Xiaoke Zhang, Qinghua Lu, Zhaofu Fei, and Paul J. Dyson. Single walled carbon nanotubes as drug delivery vehicles: Targeting doxorubicin to tumors. Biomaterials, 33(6):1689 - 1698, 2012. 
[14] Sandeep Kumar Vashist, Dan Zheng, Giorgia Pastorin, Khalid Al-Rubeaan, John H.T. Luong, and Fwu-Shan Sheu. Delivery of drugs and biomolecules using carbon nanotubes. Carbon, 49(13):4077 - 4097, 2011.

[15] Yunhui Wang, Zhongjin He, Krishna M. Gupta, Qi Shi, and Ruifeng Lu. Molecular dynamics study on water desalination through functionalized nanoporous graphene. Carbon, 116:120 - 127, 2017.

[16] Ben Corry. Designing carbon nanotube membranes for efficient water desalination. The Journal of Physical Chemistry B, 112(5):1427-1434, 2008.

[17] Rasel Das, Md Eaqub Ali, Sharifah Bee Abd Hamid, Seeram Ramakrishna, and Zaira Zaman Chowdhury. Carbon nanotube membranes for water purification: a bright future in water desalination. Desalination, 336:97-109, 2014.

[18] Ken Gethard, Ornthida Sae-Khow, and Somenath Mitra. Water desalination using carbon-nanotubeenhanced membrane distillation. ACS applied materials ES interfaces, 3(2):110-114, 2010.

[19] Ben Corry. Designing carbon nanotube membranes for efficient water desalination. The Journal of Physical Chemistry B, 112(5):1427-1434, 2008. PMID: 18163610.

[20] Matthew K Borg and Jason M Reese. Multiscale simulation of enhanced water flow in nanotubes. MRS Bulletin, 42(4):294-299, 2017.

[21] Gerhard Hummer, Jayendran C Rasaiah, and Jerzy P Noworyta. Water conduction through the hydrophobic channel of a carbon nanotube. Nature, 414(6860):188, 2001.

[22] Kerstin Falk, Felix Sedlmeier, Laurent Joly, Roland R Netz, and Lydéric Bocquet. Molecular origin of fast water transport in carbon nanotube membranes: superlubricity versus curvature dependent friction. Nano letters, 10(10):4067-4073, 2010

[23] G Algara-Siller, O Lehtinen, FC Wang, RR Nair, U Kaiser, HA Wu, AK Geim, and IV Grigorieva. Square ice in graphene nanocapillaries. Nature, 519(7544):443, 2015.

[24] Douwe Jan Bonthuis, Klaus F Rinne, Kerstin Falk, C Nadir Kaplan, Dominik Horinek, A Nihat Berker, Lydéric Bocquet, and Roland R Netz. Theory and simulations of water flow through carbon nanotubes: prospects and pitfalls. Journal of Physics: Condensed Matter, 23(18):184110, 2011.

[25] P Gallo and M Rovere. Anomalous dynamics of confined water at low hydration. Journal of Physics: Condensed Matter, 15(45):7625, 2003.

[26] Ronen Zangi and Alan E Mark. Monolayer ice. Physical review letters, 91(2):025502, 2003.

[27] Ronen Zangi. Water confined to a slab geometry: a review of recent computer simulation studies. Journal of Physics: Condensed Matter, 16(45):S5371, 2004.

[28] Ronen Zangi and Alan E Mark. Bilayer ice and alternate liquid phases of confined water. The Journal of chemical physics, 119(3):1694-1700, 2003.

[29] Nicolas Giovambattista, Peter J Rossky, and Pablo G Debenedetti. Phase transitions induced by nanoconfinement in liquid water. Physical review letters, 102(5):050603, 2009.

[30] Nicolas Giovambattista, Peter J Rossky, and Pablo G Debenedetti. Effect of pressure on the phase behavior and structure of water confined between nanoscale hydrophobic and hydrophilic plates. Physical Review E, 73(4):041604, 2006.

[31] Xin Liu, Xiulian Pan, Shanmin Zhang, Xiuwen Han, and Xinhe Bao. Diffusion of water inside carbon nanotubes studied by pulsed field gradient $\mathrm{nmr}$ spectroscopy. Langmuir, 30(27):8036-8045, 2014.

[32] Alexander I Kolesnikov, Jean-Marc Zanotti, ChunKeung Loong, Pappannan Thiyagarajan, Alexander P Moravsky, Raouf O Loutfy, and Christian J Burnham. Anomalously soft dynamics of water in a nanotube: a revelation of nanoscale confinement. Physical review letters, 93(3):035503, 2004.

[33] Jamal Hassan, G Diamantopoulos, Dirar Homouz, and George Papavassiliou. Water inside carbon nanotubes: Structure and dynamics. Nanotechnology Reviews, 5, 01 2016.

[34] Sudip Chakraborty, Hemant Kumar, Chandan Dasgupta, and Prabal K. Maiti. Confined water: Structure, dynamics, and thermodynamics. Accounts of Chemical Research, 50(9):2139-2146, 2017. PMID: 28809537.

[35] P. Hirunsit and P.B. Balbuena. Effects of confinement on water structures and dynamics:a molecular simulation study. The Journal of Physical Chemistry C, 111(4):1709-1715, 2007.

[36] Tai-De Li, Jianping Gao, Robert Szoszkiewicz, Uzi Landman, and Elisa Riedo. Structured and viscous water in subnanometer gaps. Phys. Rev. B, 75:115415, Mar 2007.

[37] Jayendran C Rasaiah, Shekhar Garde, and Gerhard Hummer. Water in nonpolar confinement: from nanotubes to proteins and beyond. Annu. Rev. Phys. Chem., 59:713-740, 2008.

[38] A. Striolo, A. A. Chialvo, K. E. Gubbins, and P. T. Cummings. Water in carbon nanotubes: Adsorption isotherms and thermodynamic properties from molecular simulation. The Journal of Chemical Physics, 122(23):234712, 2005.

[39] Leandro B Krott and Marcia C Barbosa. Anomalies in a waterlike model confined between plates. The Journal of chemical physics, 138(8):084505, 2013.

[40] Hamid Mosaddeghi, Saman Alavi, M. H. Kowsari, and Bijan Najafi. Simulations of structural and dynamic anisotropy in nano-confined water between parallel graphite plates. The Journal of Chemical Physics, 137(18):184703, 2012.

[41] P. Pugliese, M. M. Conde, M. Rovere, and P. Gallo. Freezing temperatures, ice nanotubes structures, and proton ordering of tip4p/ice water inside single wall carbon nanotubes. The Journal of Physical Chemistry B, 121(45):10371-10381, 2017. PMID: 29040802.

[42] Alessio Alexiadis and Stavros Kassinos. Molecular simulation of water in carbon nanotubes. Chemical reviews, 108(12):5014-5034, 2008.

[43] E. Sanz, C. Vega, J. L. F. Abascal, and L. G. MacDowell. Phase diagram of water from computer simulation. Phys. Rev. Lett., 92:255701, Jun 2004.

[44] William L. Jorgensen, Jayaraman Chandrasekhar, Jeffry D. Madura, Roger W. Impey, and Michael L. Klein. Comparison of simple potential functions for simulating liquid water. The Journal of Chemical Physics, 79(2):926-935, 1983. 
[45] H. J. C. Berendsen, J. P. M. Postma, W. F. van Gunsteren, and J. Hermans. Interaction Models for Water in Relation to Protein Hydration. In Bernard Pullman, editor, Intermolecular Forces, volume 14 of The Jerusalem Symposia on Quantum Chemistry and Biochemistry, pages 331-342. Springer Netherlands, 1981.

[46] Pengyu Ren and Jay W. Ponder. Polarizable atomic multipole water model for molecular mechanics simulation. The Journal of Physical Chemistry B, 107(24):5933-5947, 2003.

[47] Jose LF Abascal and Carlos Vega. A general purpose model for the condensed phases of water: Tip4p/2005. The Journal of chemical physics, 123(23):234505, 2005.

[48] H. M. Köhler, R. J. Bordin, da Silva B. L., and C. M. Barbosa. Breakdown of the stokes-einstein water transport through narrow hydrophobic nanotubes. Physical Chemistry Chemical Physics, 19:12921-12927, 2017.

[49] Jordi Mart', Carles Calero, and Giancarlo Franzese. Structure and dynamics of water at carbon-based interfaces. Entropy, 19(3):135, 2017.

[50] Pradeep Kumar, Sergey V Buldyrev, Francis W Starr, Nicolas Giovambattista, and H Eugene Stanley. Thermodynamics, structure, and dynamics of water confined between hydrophobic plates. Physical Review E, 72(5):051503, 2005.

[51] Alessio Alexiadis and Stavros Kassinos. The density of water in carbon nanotubes. Chemical Engineering Science, 63(8):2047-2056, 2008.

[52] Alessio Alexiadis and Stavros Kassinos. Self-diffusivity, hydrogen bonding and density of different water models in carbon nanotubes. Molecular Simulation, 34(7):671678, 2008.

[53] Brad A Bauer, Shuching Ou, Sandeep Patel, and Karthik Siva. Dynamics and energetics of hydrophobically confined water. Physical Review E, 85(5):051506, 2012.

[54] Yong-gang Zheng, Hong-fei Ye, Zhong-qiang Zhang, and Hong-wu Zhang. Water diffusion inside carbon nanotubes: mutual effects of surface and confinement. Physical Chemistry Chemical Physics, 14(2):964-971, 2012.

[55] Ioannis N. Tsimpanogiannis, Othonas A. Moultos, Lus F. M. Franco, Marcelle B. de M. Spera, Mt Erds, and Ioannis G. Economou. Self-diffusion coefficient of bulk and confined water: a critical review of classical molecular simulation studies. Molecular Simulation, 0(0):1-29, 2018.

[56] Simon Gravelle, Christophe Ybert, Lydéric Bocquet, and Laurent Joly. Anomalous capillary filling and wettability reversal in nanochannels. Phys. Rev. E, 93(3):033123, mar 2016.

[57] Li Fu, Samy Merabia, and Laurent Joly. Understanding Fast and Robust Thermo-osmotic Flows through Carbon Nanotube Membranes: Thermodynamics Meets Hydrodynamics. J. Phys. Chem. Lett., 9(8):2086-2092, apr 2018.

[58] L Liu and GN Patey. Simulations of water transport through carbon nanotubes: how different water models influence the conduction rate. The Journal of chemical physics, 141(18):18C518, 2014.

[59] Yoshimichi Nakamura and Takahisa Ohno. Structure of water confined inside carbon nanotubes and water mod- els. Materials Chemistry and Physics, 132(2-3):682-687, 2012.

[60] Christa Guse and Reinhard Hentschke. Simulation study of structural, transport, and thermodynamic properties of tip $4 \mathrm{p} / 2005$ water in single-walled carbon nanotubes. The Journal of Physical Chemistry B, 116(2):751-762, 2012. PMID: 22171918.

[61] L. Liu and G. N. Patey. Simulated conduction rates of water through a $(6,6)$ carbon nanotube strongly depend on bulk properties of the model employed. J. Chem. Phys., 144(18):184502, 2016.

[62] Berk Hess, Carsten Kutzner, David Van Der Spoel, and Erik Lindahl. Gromacs 4: algorithms for highly efficient, load-balanced, and scalable molecular simulation. Journal of chemical theory and computation, 4(3):435-447, 2008.

[63] Giovanni Bussi, Davide Donadio, and Michele Parrinello. Canonical sampling through velocity rescaling. The Journal of chemical physics, 126(1):014101, 2007.

[64] Stefano Bernardi, BD Todd, and Debra J Searles. Thermostating highly confined fluids. The Journal of chemical physics, 132(24):244706, 2010.

[65] Jeetu S. Babu and Sarith P. Sathian. The role of activation energy and reduced viscosity on the enhancement of water flow through carbon nanotubes. The Journal of Chemical Physics, 134(19):194509, 2011.

[66] Sridhar Kumar Kannam, B. D. Todd, J. S. Hansen, and Peter J. Daivis. Slip length of water on graphene: Limitations of non-equilibrium molecular dynamics simulations. The Journal of Chemical Physics, 136(2):024705, 2012.

[67] Jiabo Tao, Xianyu Song, Wei Chen, Shuangliang Zhao, and Honglai Liu. Thermostat effect on water transport dynamics across cnt membranes. Molecular Simulation, pages 1-7, 2018.

[68] Berk Hess. Determining the shear viscosity of model liquids from molecular dynamics simulations. The Journal of Chemical Physics, 116(1):209-217, 2002.

[69] Ulrich Essmann, Lalith Perera, Max L. Berkowitz, Tom Darden, Hsing Lee, and Lee G. Pedersen. A smooth particle mesh ewald method. The Journal of Chemical Physics, 103(19):8577-8593, 1995.

[70] William L Jorgensen and Julian Tirado-Rives. The opls [optimized potentials for liquid simulations] potential functions for proteins, energy minimizations for crystals of cyclic peptides and crambin. Journal of the American Chemical Society, 110(6):1657-1666, 1988.

[71] Ian Moskowitz, Mark A Snyder, and Jeetain Mittal. Water transport through functionalized nanotubes with tunable hydrophobicity. The Journal of chemical physics, 141(18):18C532, 2014.

[72] Aparna Waghe, Jayendran C Rasaiah, and Gerhard Hummer. Filling and emptying kinetics of carbon nanotubes in water. The Journal of chemical physics, 117(23):10789-10795, 2002.

[73] William Humphrey, Andrew Dalke, and Klaus Schulten. Vmd: visual molecular dynamics. Journal of molecular graphics, 14(1):33-38, 1996.

[74] John A Thomas and Alan JH McGaughey. Water flow in carbon nanotubes: transition to subcontinuum transport. Physical Review Letters, 102(18):184502, 2009.

[75] R Kumar, JR Schmidt, and JL Skinner. Hydrogen 
bonding definitions and dynamics in liquid water. The Journal of chemical physics, 126(20):05B611, 2007.

[76] Fengchao Wang and Yapu Zhao. The unique properties of the solid-like confined liquid films: A large scale molecular dynamics simulation approach. Acta Mechanica Solida Sinica, 24(2):101-116, Apr 2011.

[77] Peter Daivis and Billy Todd. Challenges in nanofluidicsbeyond navier-stokes at the molecular scale. Processes, 6(9):144, 2018.

[78] Hai Hoang and Guillaume Galliero. Local viscosity of a fluid confined in a narrow pore. Physical Review E, 86(2):021202, 2012.

[79] Jesper S Hansen, Jeppe C Dyre, Peter Daivis, Billy D Todd, and Henrik Bruus. Continuum nanofluidics. Langmuir, 31(49):13275-13289, 2015.

[80] M. Morciano, M. Fasano, A. Nold, C. Braga, P. Yatsyshin, D. N. Sibley, B. D. Goddard, E. Chiavazzo, P. Asinari, and S. Kalliadasis. Nonequilibrium molecular dynamics simulations of nanoconfined fluids at solidliquid interfaces. The Journal of Chemical Physics, 146(24):244507, 2017.

[81] Burkhard Dünweg and Kurt Kremer. Molecular dynamics simulation of a polymer chain in solution. The Journal of chemical physics, 99(9):6983-6997, 1993.

[82] In-Chul Yeh and Gerhard Hummer. System-size dependence of diffusion coefficients and viscosities from molecular dynamics simulations with periodic boundary conditions. The Journal of Physical Chemistry B, 108(40):15873-15879, 2004.

[83] Sami Tazi, Alexandru Boţan, Mathieu Salanne, Virginie Marry, Pierre Turq, and Benjamin Rotenberg. Diffusion coefficient and shear viscosity of rigid water models. Journal of Physics: Condensed Matter, 24(28):284117, 2012.

[84] Martin Vögele and Gerhard Hummer. Divergent diffusion coefficients in simulations of fluids and lipid membranes. The Journal of Physical Chemistry B, 120(33):8722-8732, 2016.

[85] Pauline Simonnin, Beno^t Noetinger, Carlos NietoDraghi, Virginie Marry, and Benjamin Rotenberg. Diffusion under Confinement: Hydrodynamic Finite-Size Effects in Simulation. J. Chem. Theory Comput., 13(6):2881-2889, 2017.

[86] John Happel and Howard Brenner. Low Reynolds number hydrodynamics: with special applications to particulate media, volume 1. Springer Science \& Business Media, 2012.

[87] A Saugey, L Joly, C Ybert, J L Barrat, and L Bocquet. Diffusion in pores and its dependence on boundary conditions. J. Phys. Condens. Matter, 17(49):S4075-S4090, 2005.

[88] Eric Lauga and Todd M. Squires. Brownian motion near a partial-slip boundary: A local probe of the noslip condition. Phys. Fluids, 17(10):103102, 2005.

[89] L Joly, C Ybert, and L Bocquet. Probing the Nanohydrodynamics at Liquid-Solid Interfaces Using Thermal Motion. Phys. Rev. Lett., 96(4):046101, feb 2006.

[90] François Detcheverry and Lydéric Bocquet. Thermal Fluctuations in Nanofluidic Transport. Phys. Rev. Lett., 109(2):024501, 2012.

[91] François Detcheverry and Lydéric Bocquet. Thermal fluctuations of hydrodynamic flows in nanochannels.
Phys. Rev. E, 88(1):012106, jul 2013.

[92] A. Einstein. Über die von der molekularkinetischen Theorie der Wärme geforderte Bewegung von in ruhenden Flüssigkeiten suspendierten Teilchen. Ann. Phys., 322(8):549-560, 1905.

[93] Jason K Holt. Fast mass transport through sub2-nanometer carbon nanotubes. Science (80-. )., 312(5776):1034-1037, 2006.

[94] Eleonora Secchi, Sophie Marbach, Antoine Niguès, Derek Stein, Alessandro Siria, and Lydéric Bocquet. Massive radius-dependent flow slippage in carbon nanotubes. Nature, 537(7619):210-213, sep 2016.

[95] Sridhar Kumar Kannam, B D Todd, J S Hansen, and Peter J Daivis. How fast does water flow in carbon nanotubes? J. Chem. Phys., 138(9):094701, 2013.

[96] Kerstin Falk, Felix Sedlmeier, Laurent Joly, Roland R Netz, and Lydéric Bocquet. Molecular origin of fast water transport in carbon nanotube membranes: superlubricity versus curvature dependent friction. Nano Lett., 10(10):4067-4073, 2010.

[97] Emmanuel Guillaud, Laurent Joly, Dominique de Ligny, and Samy Merabia. Assessment of elastic models in supercooled water: A molecular dynamics study with the TIP4P/2005f force field. J. Chem. Phys., 147(1):014504, 2017.

[98] Pablo Montero de Hijes, Eduardo Sanz, Laurent Joly, Chantal Valeriani, and Frédéric Caupin. Viscosity and self-diffusion of supercooled and stretched water from molecular dynamics simulations. arXiv preprint arXiv:1805.11957, 2018.

[99] Zhongzhong Cui, Dechang Li, and Baohua Ji. Effect of curvature on the behaviors of confined water layers. International Journal of Applied Mechanics, 7(06):1550084, 2015.

[100] Thomas Werder, Jens H Walther, Richard L Jaffe, Timur Halicioglu, Flavio Noca, and Petros Koumoutsakos. Molecular dynamics simulation of contact angles of water droplets in carbon nanotubes. Nano Letters, 1(12):697-702, 2001.

[101] LM Alarcón, DC Malaspina, EP Schulz, MA Frechero, and GA Appignanesi. Structure and orientation of water molecules at model hydrophobic surfaces with curvature: From graphene sheets to carbon nanotubes and fullerenes. Chemical Physics, 388(1-3):47-56, 2011.

[102] L Fumagalli, A Esfandiar, R Fabregas, S Hu, P Ares, A Janardanan, Q Yang, mB Radha, T Taniguchi, $\mathrm{K}$ Watanabe, et al. Anomalously low dielectric constant of confined water. Science, 360(6395):1339-1342, 2018.

[103] Xiaoxia Cai, Wen Jun Xie, Ying Yang, Zhuoran Long, Jun Zhang, Zhuoran Qiao, Lijiang Yang, and Yi Qin Gao. Structure of water confined between two parallel graphene plates. The Journal of Chemical Physics, 150(12):124703, 2019.

[104] S Chen, AP Draude, AXC Nie, HP Fang, NR Walet, Shiwu Gao, and JC Li. Effect of layered water structures on the anomalous transport through nanoscale graphene channels. Journal of Physics Communications, 2(8):085015, 2018.

[105] James Dix, Leo Lue, and Paola Carbone. Why different water models predict different structures under $2 \mathrm{~d}$ confinement. Journal of computational chemistry, 
39(25):2051-2059, 2018.

[106] Guillaume Lamour, Ahmed Hamraoui, Andrii Buvailo, Yangjun Xing, Sean Keuleyan, Vivek Prakash, Ali Eftekhari-Bafrooei, and Eric Borguet. Contact angle measurements using a simplified experimental setup. Journal of chemical education, 87(12):1403-1407, 2010. 